\title{
Equal Gain Block Decomposition Methods for Multiuser MIMO Networks
}

\author{
Insoo Hwang', Inseok Kang ${ }^{2}$, Intae Hwang ${ }^{3}$, and Cheolwoo You ${ }^{2 *}$ \\ ${ }^{1}$ Qualcomm Research, San Diego, CA 92121-1608, USA \\ [e-mail: insooh@qualcomm.com] \\ ${ }^{2}$ Department of Information and Communications Engineering, Myongji University \\ Yongin, Gyeonggi-do, 17058 Republic of Korea \\ [e-mail: kang060215@gmail.com, cwyou@mju.ac.kr] \\ ${ }^{3}$ Department of Electronics and Computer Engineering, Chonnam National University \\ Yongbong-dong, Buk-gu, Gwangju 500-757, Republic of Korea \\ [hit@chonnam.ac.kr] \\ ${ }^{*}$ Corresponding author: Cheolwoo You
}

Received December 6, 2020; accepted December 19, 2020; published March 31, 2021

\begin{abstract}
In this paper, we propose a new joint precoder and postcoder design strategy to support multiple streams per user in multiuser multiple-input multiple-output (MIMO) systems. We propose two step precoding strategies using equal channel gain decomposition and block diagonalization at the transmitter. With the proposed precoder, the multiuser MIMO channel can be decomposed into multiple parallel channels with equal channel gain per user. After applying receive postcoder which is generated and sent by the transmitter, we can use ML based decoder per stream to achieve full receive diversity. Achievable sum rate bound and diversity performance of the proposed algorithm are presented with feedback signaling design and quantitative complexity analysis. Simulation results show that the proposed algorithm asymptotically approaches to the sum rate capacity of the MIMO broadcast channel while maintaining full diversity order.
\end{abstract}

Keywords: Precoder, Postcoder, MIMO, Multiuser, Full Diversity Order

This work was supported by Institute of Information \& communications Technology Planning \& Evaluation (IITP) grant funded by the Korea government(MSIT) (No.2020-0-00994, Development of autonomous VR and AR content generation technology reflecting usage environment). This work was also supported by the National Research Foundation of Korea (NRF) grant funded by the Korea government (MSIP) (No. 2019R1H1A2077966). 


\section{Introduction}

Recent academic and industry interest has shifted from point-to-point multiple-input multiple- output (MIMO) channels to MIMO broadcast channels [1]. In the MIMO broadcast channel, multiple users can be supported simultaneously by sharing spatial resources. The sum capacity region of the MIMO broadcast channel has been extensively studied [2-12]. The optimal transmit strategy that achieves the MIMO broadcast channel capacity is inspired by dirty-paper coding (DPC) [2], but information-theoretic DPC does not give direct realization of practical transmit strategies.

As a practical and simple solution, linear precoding methods such as zero-forcing (ZF) and block diagonalization (BD) have been suggested [13-17]. ZF is based on the channel inversion, but it suffers from excessive transmit power loss due to the weakest stream $[13,18]$. BD is a generalization of $\mathrm{ZF}$ that supports multiple streams per user. BD makes the effective channel block diagonal; hence multiuser interference is cancelled at the transmitter, but the interstream interference is remained for the decoder processing. Those linear precoding algorithms achieve sum rate throughput close to DPC in many scenarios with far reduced complexity. But the diversity order of those linear techniques is one, resulting in huge error rate performance degradation in higher SNR regime.

To compensate for low diversity order in linear precoding methods, nonlinear precoding or decoding methods can be used to give more diversity order. Nonlinear precoding methods such as $[19,20]$, however, are still too complex compared to linear precoding methods. Complexity issues for the nonlinear decoder have been solved by recent developments on reduced complexity maximum likelihood (ML) decoders [21-26]. As the number of streams per user is smaller than the number of transmit antennas, the usage of nonlinear technique in decoder is advantageous than using nonlinear technique for precoding. A proper joint design of the linear precoder and the nonlinear decoder for the multiuser MIMO systems is, therefore, a promising practical solution to achieve good throughput and diversity order simultaneously.

One most challenging problem of nonlinear decoder in the multiuser MIMO systems is to obtain a post-processing signal to interference plus noise ratio (SINR). Obtaining exact postprocessing SINR value per stream is important since the obtained SINR plays a key role for the link adaptation. Link adaptation using adaptive modulation and coding (AMC) mechanism for multiuser MIMO uses the effective SINR as an indicator of the link quality. Unlike to the linear receiver where the SINR value can be easily computed, there is no known explicit way to calculate accurate per stream effective SINR after nonlinear decoding, when the SINR level per stream differs from each other. Due to this limitation, one cannot fully take advantage of both linear-based simple transmit strategy and optimal decoding strategy in multiuser MIMO systems so far.

In this paper, we propose a new precoding and postcoding algorithm for multiuser MIMO with ML based nonlinear decoder, when multiple streams are supported per user. We propose a precoding method that makes the effective channel to have equal diagonal element, as in [27, 28], to obtain a precise post-processing SINR. The equal channel gain decomposition such as uniform channel decomposition (UCD) and geometric mean decomposition (GMD) in [27, 28] were originally suggested for single user MIMO and optimized for the minimum mean square error (MMSE) with successive interference cancellation or ZF-DPC based decoder. We suggest a two-step precoding method using $\mathrm{BD}$ and equal channel gain decomposition to eliminate multiuser diversity and to derive SINR easily. Using the proposed two-step precoding at the transmitter, each user independently uses the ML decoder to get full diversity. The proposed algorithm also generates exact per stream SINR to apply for link adaptation. 
The main contributions of this paper are summarized as follows.

- We propose a new joint precoding and postcoding method for multiuser MIMO with multiple stream support per user. The proposed algorithm achieves good sum rate and full diversity gain; hence it shows good error rate performance as well as good throughput performance. Both analytical derivation and simulation results of our proposed algorithm are presented.

- Signaling strategies is presented, reducing feedback overhead while minimizing feedback mismatch. We also present the issues when generating link curve using ML decoder and effective SINR for link adaptation. Using the proposed algorithm, the feedback generation mechanism is simplified and the accuracy of the effective SINR for link adaptation is increased.

- We compare our proposed algorithm to several previously proposed single user and multiuser MIMO algorithms. Comparison includes simple singular value decomposition (SVD) based channel decomposition, Orthogonal Beamforming (OBF) [14], BD [16] and UCD [28]. The comparison includes precoder design, decoding method, achievable sum rate, feedback requirement as well as complexity.

The outline of this paper is as follows. In Section 2, we introduce the system model for the MIMO broadcast channel and introduce basic algorithms of BD and UCD. In Section 2, we propose a new precoder and postcoder for the nonlinear decoder. Sum rate analysis, diversity gain analysis and feedback strategy are also presented in the same section. Simulation results and comparison with previously proposed methods are given in Section 4, followed by conclusions and future works in Section 5.

\section{System Model and Prior Works}

In this section, we introduce the system model and prior works related to this paper. A brief introduction on BD, GMD and UCD is provided in the following few subsections. Throughout this paper, we use upper boldface $\mathbf{A}$ and lower boldface $\mathbf{a}$ as a matrix and a vector, respectively. We denote the inverse, transpose, Hermitian of matrix $\mathbf{A}$ by $\mathbf{A}^{-1}, \mathbf{A}^{\mathrm{T}}$ and $\mathbf{A}^{*}$, respectively. $\|a\|$ denotes vector 2-norm, unless otherwise stated.

\subsection{System Model}

Consider a MIMO broadcast channel with a single transmitter and $U$ users, as shown in Fig. 1. The transmitter has $N_{t}$ antennas and the $k$ th user has $N_{r, k}$ receive antennas, hence the sum of all the receive antennas are $N_{r}=\sum_{k=1}^{U} N_{r, k}$. Without loss of generality, we let $N_{r}=N_{t}$ for simplicity. Let the channel between the transmitter and $k$ th user is $N_{r, k} \times N_{t}$ matrix $\mathbf{H}_{k}$, hence the aggregated channel for all $U$ users can be written as $\boldsymbol{H}=\left[\begin{array}{lll}\boldsymbol{H}_{1}^{T} & \cdots & \boldsymbol{H}_{U}^{T}\end{array}\right]^{T}$. Let the transmit symbol vector for $k$ th user is $\mathbf{x}_{k}$ and its corresponding transmit precoding matrix is $\boldsymbol{F}=$ $\left[\begin{array}{lll}\boldsymbol{F}_{1} & \cdots & \boldsymbol{F}_{U}\end{array}\right]$, with size $N_{t} \times N_{t}$. Let the $N_{r, k} \times N_{t}$ receive postcoding matrix $\mathbf{W}_{k}$, then the received vector at the $k$ th user is given by

$$
\mathbf{y}_{k}=\mathbf{W}_{k}^{*} \mathbf{H}_{k} \mathbf{F} \mathbf{x}+\mathbf{n}_{k}=\mathbf{W}_{k}^{*} \mathbf{H}_{k} \mathbf{F}_{k} \mathbf{x}_{k}+\sum_{j=1, j \neq k}^{U} \mathbf{W}_{k}^{*} \mathbf{H}_{k} \mathbf{F}_{j} \mathbf{x}_{j}+\mathbf{n}_{k}
$$


where $\boldsymbol{x}=\sum_{k=1}^{U} \boldsymbol{x}_{k}$ is the $N_{t} \times 1$ transmit vector that each of the component is drawn from finite complex alphabet $C$. The overall received vector over all the users is $\boldsymbol{y}=\left[\begin{array}{lll}\boldsymbol{y}_{1}^{T} & \cdots & \boldsymbol{y}_{U}^{T}\end{array}\right]^{T}$. The $N_{r, k} \times 1$ vector $\mathbf{n}$ is an additive complex Gaussian noise vector in the $k$ th user, with mean zero and covariance matrix $\sigma_{n} \boldsymbol{I}_{r, k} . L_{k}$ is the number of streams sent to the user $k$, hence $\sum_{k=1}^{U} L_{k}=N_{t}$. We assume that $\mathbf{x}$ is normalized such that the average transmit power is $P_{T}$.

The goal of this paper is to find the precoding matrix $\mathbf{F}$ and decoder combining matrix $\mathbf{W}_{k}$ $(k=1, \cdots, U)$ to minimize multiuser interference $\sum_{j=1, j \neq k}^{U} \boldsymbol{W}_{k}^{*} \boldsymbol{H}_{k} \boldsymbol{F}_{j} \boldsymbol{x}_{j}$ in (1) and maximize sum throughput of the multiuser MIMO link.

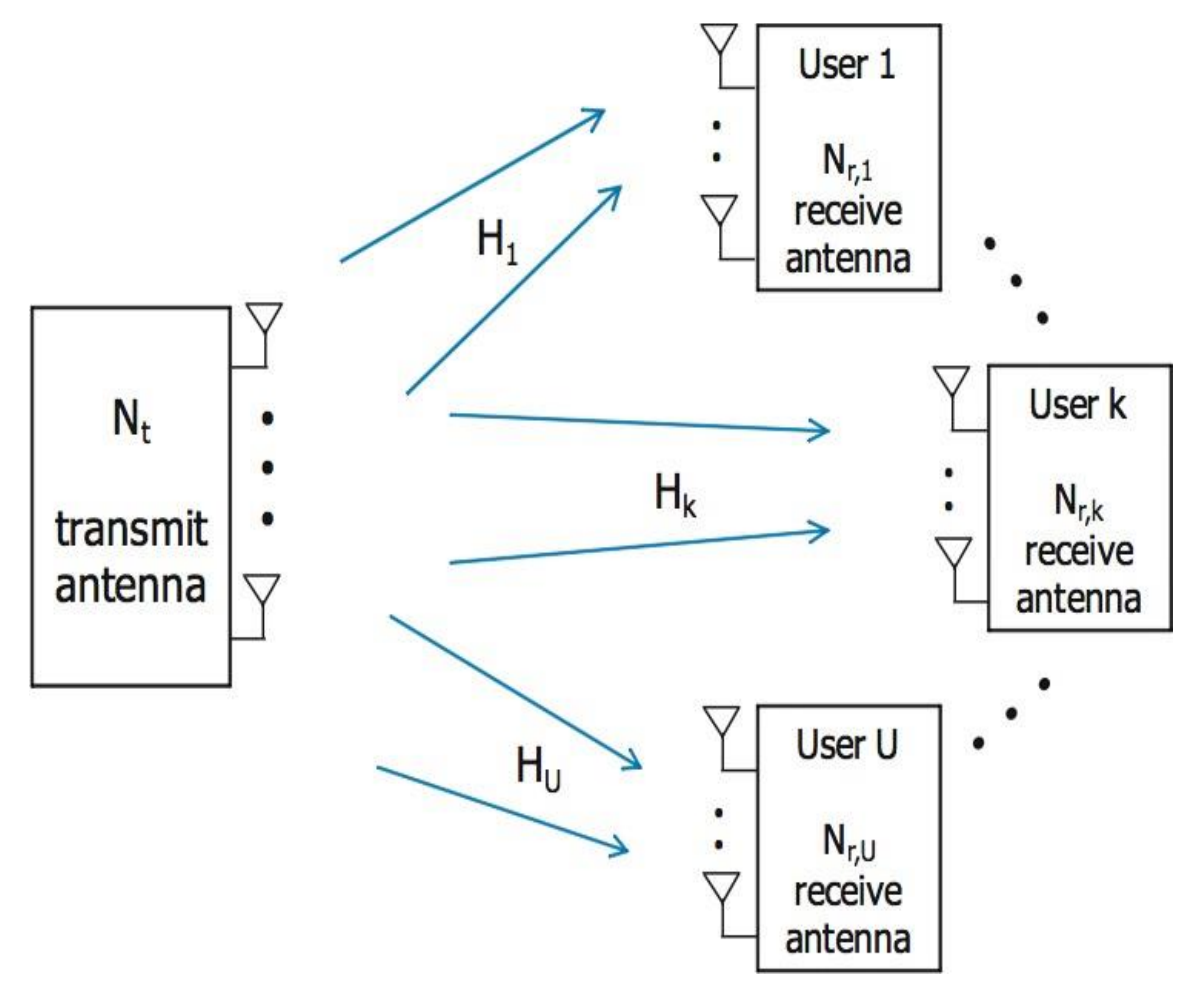

Fig. 1. System Model

\subsection{Block Diagonalization (BD)}

$\mathrm{BD}$ is a generalization of ZF linear precoding when the number of streams per user is more than one. Linear preprocessing is performed at the transmitter by multiplying transmit beamformer $\mathbf{F}$ to the transmit signal. Receive postcoder $\mathbf{W}_{k}$ for the $k$ th user is used to overcome dimensionality constraint. To eliminate multiuser interference, BD imposes the zerointerference constraint, $\mathbf{H}_{i} \mathbf{F}_{j}=0$ for $i \neq j$. The zero-interference constraint between users forces $\mathbf{F}_{j}$ to lie in the null space of $\widetilde{\mathbf{H}}_{j}=\left[\mathbf{H}_{1}^{T} \cdots \mathbf{H}_{j-1}^{T} \mathbf{H}_{j+1}^{T} \cdots \mathbf{H}_{U}^{T}\right]^{T}=\widetilde{\mathbf{U}}_{j} \widetilde{\Sigma}_{j}\left[\widetilde{\mathbf{V}}_{j}^{1} \widetilde{\mathbf{V}}_{j}^{0}\right]^{*}$ where $\widetilde{\mathbf{V}}_{j}^{1}$ and $\widetilde{\mathbf{V}}_{j}^{0}$ are right singular matrices corresponding nonzero and zero singular values of $\widetilde{\mathbf{H}}_{j}$, respectively. Multiplying $\widetilde{\mathbf{V}}_{j}^{0}$ to the $j$ th user's channel matrix $\mathbf{H}_{j}$ and applying singular value decomposition (SVD) yields 


$$
\mathbf{H}_{j} \widetilde{\mathbf{V}}_{j}^{0}=\mathbf{U}_{j}\left[\begin{array}{cc}
\Sigma_{j} & \mathbf{0} \\
\mathbf{0} & \mathbf{0}
\end{array}\right]\left[\begin{array}{ll}
\mathbf{V}_{j}^{1} & \mathbf{V}_{j}^{0}
\end{array}\right]^{*}
$$

The product of $\widetilde{\boldsymbol{V}}_{j}^{0}$ and $\boldsymbol{V}_{j}^{1}$ make the channel $\mathbf{H}_{j}$ free from the multiuser interference since the right singular matrix $\boldsymbol{V}_{j}^{1}$ corresponding nonzero singular vector is obtained by the multiplication of $\widetilde{\mathbf{V}}_{j}^{0}$ to the channel. Then the BD precoder is obtained as $\mathbf{F}=$ $\left[\widetilde{\mathbf{V}}_{1}^{0} \boldsymbol{V}_{1}^{1} \cdots \widetilde{\mathbf{V}}_{U}^{0} \boldsymbol{V}_{U}^{1}\right] \Lambda^{1 / 2}$, where $\boldsymbol{\Lambda}$ is power scaling matrix. The first $L_{j}$ column of the left singular matrix $\mathbf{U}_{j}$ is used for decoder combining. The above process can be seen as a decomposition technique of $N_{r} \times N_{t}$ physical channel into $U$ parallel $\left(N_{r}-(U-1) N_{t}\right) \times N_{t}$ channels [29].

\subsection{Uniform Channel Decomposition (UCD)}

UCD is a matrix decomposition method that makes a MIMO channel into multiple subchannels with the same singular values [28]. It can be considered as a modified QR decomposition since the output is a multiplication of upper triangular matrix and unitary matrices. The precoder for UCD is given as $\mathbf{V} \boldsymbol{\Phi} \boldsymbol{\Omega}^{*}$, where $\boldsymbol{\Phi}$ is power loading diagonal matrix as the water-filling solution $\Phi \triangleq\left(\mu-\alpha \Lambda^{-2}\right)_{+}^{1 / 2}\left([a]_{+} \triangleq \max \{0, a\}\right)$ with sum power constraint $\operatorname{tr}\left\{\boldsymbol{\Phi}^{2}\right\}=P_{T}$ [30]. $\boldsymbol{\Omega}$ is $N_{t} \times N_{t}$ unitary matrix to be derived by UCD process and $\mathbf{V}$ is the left singular matrix in SVD. Using the extended channel matrix $\overrightarrow{\boldsymbol{H}}=$ $[\boldsymbol{H} \sqrt{\alpha} \boldsymbol{I}]^{T}$, where $\alpha=1 / N_{t}$ is the inverse of SNR, the channel can be decomposed as

$$
\overline{\boldsymbol{H}}_{U C D} \triangleq\left[\begin{array}{c}
\boldsymbol{H} \boldsymbol{F} \\
\sqrt{\alpha} \boldsymbol{I}_{N_{t}}
\end{array}\right]=\left(\begin{array}{c}
\boldsymbol{U} \boldsymbol{\Lambda} \boldsymbol{\Phi} \boldsymbol{\Omega}^{*} \\
\sqrt{\alpha} \boldsymbol{I}_{N_{t}}
\end{array}\right)=\boldsymbol{Q} \boldsymbol{R} \boldsymbol{P}^{*}
$$

where $\mathbf{Q}$ is $\left(N_{r}+N_{t}\right) \times N_{t}, \mathbf{P}$ is $N_{t} \times N_{t}$ unitary matrices. The $N_{t} \times N_{t}$ upper triangular matrix $\mathbf{R}$ with equal singular values $\bar{r}=\bar{\sigma}=\left(\prod_{k=1}^{N_{t}} \sqrt{\sigma_{k}^{2}+\alpha}\right)^{1 / N_{t}}$ is achieved by symmetric permutation and orthogonalization using the a series of $2 \times 2$ Givens rotation process. We note that $\Omega$ is a multiplication of power loading unitary matrices derived from each Givens rotation process as $\boldsymbol{\Omega}=\mathbf{P}_{1} \mathbf{P}_{2} \cdots \mathbf{P}_{N_{t}-1}=\mathbf{P}$. Define $\mathbf{Q}=\left[\widetilde{\mathbf{Q}}_{u} \widetilde{\mathbf{Q}}_{l}\right]^{T}$, where $N_{r} \times N_{t}$ matrix $\widetilde{\mathbf{Q}}_{u}$ is the first $N_{r}$ rows of $\mathbf{Q}$ and $N_{t} \times N_{t}$ matrix $\widetilde{\mathbf{Q}}_{l}$ is the last $N_{t}$ rows of $\mathbf{Q}$. Applying the transmit precoder $\mathbf{F}=\mathbf{V} \boldsymbol{\Phi} \boldsymbol{\Omega}$ and the decoder filter $\widetilde{\mathbf{Q}}_{u}^{*}$, the output signal can be expressed as

$$
\tilde{\mathbf{y}}=\widetilde{\mathbf{Q}}_{u}^{*} \mathbf{H V} \Phi \mathbf{P x}+\widetilde{\mathbf{Q}}_{u}^{*} \mathbf{n}=\mathbf{R x}-\sqrt{\alpha} \widetilde{\mathbf{Q}}_{l}^{*} \mathbf{P x}+\widetilde{\mathbf{Q}}_{u}^{*} \mathbf{n}
$$

The transmitted vector $\mathbf{x}$ can be detected by the MMSE with successive interference canceler or Tomlinson-Harashima Precoding (THP). GMD in [27] is a special case of UCD, with $\boldsymbol{\Phi}=\boldsymbol{I}_{N_{t}}$ and $\alpha=0$.

\subsection{Block Diagonalizable UCD (BD-UCD)}

Concatenation of UCD with block diagonalizable matrix are suggested in [31-33]. The main purpose of $[31,32]$ is to make each user have equal rates for its data streams, by using block decomposed GMD and UCD. They allow each user to apply equal rate encoding on its own subchannels. The main purpose of $[31,32]$, however, is to construct a decoder filter that has a 
block-diagonal form. To nullify multiuser interference in [31,32], a universal postcoder of size $U \times N_{t}$ should be given to all the users. The method to eliminate multiuser interference at the receive side is nothing but $\mathrm{ZF}$ decoder. It differs from the BD in 2.2, which is based on user allocation to the null space of the previously chosen user. In [33], block diagonalized UCD is used only for single user point-to-point channel. It simply pairs two eigenvalues with smaller and larger one by stream ordering and user ML decoding per each pair.

\section{A New Two Step Precoding Algorithm}

In this section, a new linear transmit-receive joint optimization method is presented. We propose a two-step precoding method based on equal channel gain decomposition with blockwise diagonalization to simplify decoding and generating feedback. Sum rate capacity analysis, diversity analysis and comparison with various closed-loop MIMO schemes are also introduced in this section, followed by numerical simulation results in Section 4.

\subsection{Transmit Strategies}

The goal of the precoder design is to find an appropriate unitary precoding matrix $\mathbf{F}$ and decoder combining matrix $\mathbf{W}_{k}$ to make the effective channel $\overline{\mathbf{H}}$ block diagonal and equal channel gain within each user $k$, as

$$
\mathbf{y}_{k}=\mathbf{W}_{k}^{*} \widetilde{\mathbf{H}}_{k} \mathbf{F x}+\mathbf{W}_{k}^{*} \mathbf{n}_{k} \triangleq \overline{\mathbf{H}}_{k} \mathbf{x}+\overline{\mathbf{n}}_{k}
$$

where $\widetilde{\mathbf{H}}_{k}$ is the effective channel with zero multiuser interference. To enforce zero interference criterion between users, a nulling matrix at user $k$ using $\mathbf{F}_{B D}=\left[\widetilde{\mathbf{V}}_{1}^{0} \boldsymbol{V}_{1}^{1} \cdots \widetilde{\mathbf{V}}_{U}^{0} \boldsymbol{V}_{U}^{1}\right]^{T}$ and postcoding matrix $\mathbf{U}_{k}$ can be obtained using block diagonalization described in Section 2.2. Then the effective channel for the $k$ th user is

$$
\widetilde{\mathbf{H}}_{k}=\mathbf{U}_{k} \mathbf{H}_{k} \mathbf{F}_{B D} .
$$

The second precoder $\boldsymbol{F}=\left[\begin{array}{lll}\boldsymbol{F}_{1} & \cdots & \boldsymbol{F}_{U}\end{array}\right]$ makes the effective channel per user have equal diagonalization, without hurting the zero-interference criterion. It is straightforward that $\mathbf{W}_{j}^{*} \widetilde{\mathbf{H}}_{j} \mathbf{F}_{k}=0$ yields $\boldsymbol{W}_{j}^{*} \boldsymbol{U}_{j} \boldsymbol{H}_{j} \boldsymbol{F}_{B D} \boldsymbol{F}_{k}=0$ when $j \neq k$. By combining (5) and (6), we have the input-output relations for the $k$ th user as

$$
\boldsymbol{y}_{k}=\overline{\boldsymbol{H}}_{k} \boldsymbol{x}+\overline{\boldsymbol{n}}_{k}=\boldsymbol{W}_{k}^{*} \boldsymbol{U}_{k} \boldsymbol{H}_{k} \boldsymbol{F}_{B D} \boldsymbol{F} \boldsymbol{x}+\overline{\boldsymbol{n}}_{k} .
$$

The aggregated effective channel can be written as $\overline{\boldsymbol{H}}=\left[\begin{array}{lll}\overline{\boldsymbol{H}}_{1}^{T} & \cdots & \overline{\boldsymbol{H}}_{U_{U}}^{T}\end{array}\right]^{T}$, where $\overline{\boldsymbol{H}}_{k}$ is the $k$ th user's effective subchannel with equal channel gain with rank $L_{k}$.

To compute the second precoder and postcoder for the $k$ th user, we use SVD of $\widetilde{\mathbf{H}}_{k}=$ $\widetilde{\mathbf{U}}_{k} \widetilde{\Lambda}_{k} \widetilde{\mathbf{V}}_{k}$ and define the precoder $\mathbf{F}$ as

$$
\mathbf{F}_{k}=\widetilde{\mathbf{V}}_{k} \Phi_{k} \mathbf{P}_{k}
$$

where $\boldsymbol{\Phi}_{k}$ is the $k$-th user's power loading matrix derived by the waterfilling solution of $\widetilde{\mathbf{H}}_{k}$ [30] and $\mathbf{P}_{k}$ is the precoding matrix explained in Section 2.3. To prevent error propagation and change of second order channel statistics, we assume $\mathbf{P}_{k}$ be a unitary matrix. Let us define 
$L_{k} \times L_{k}$ diagonal matrix $\Sigma_{k} \triangleq \widetilde{\Lambda}_{k} \boldsymbol{\Phi}_{k}=\operatorname{diag}\left\{\sigma_{1}, \sigma_{2}, \cdots, \sigma_{L_{k}}\right\}$. The number of possible streams for the $k$ th user $L_{k}$ is upper bounded by the dimension of the matrix $\widetilde{\mathbf{H}}_{k}$, hence $L_{k} \leq$ $N_{r, k} . L_{k}$ is also lower bounded by the minimum numbers of streams for the $k$ th user to have at least one stream, $L_{k}>\operatorname{rank}\left(\mathbf{H}_{k}\right)+\operatorname{nullity}\left(\widetilde{\mathbf{H}}_{k}\right)-N_{t}$. To have more degree of freedom and consequently more diversity gain at the decoder, larger $L_{k}$ with larger eigenvalues is beneficial. Otherwise, if all of $U$ users have just one stream, block diagonalization simply become an inverse of the aggregated channel matrix, resulting in large transmit power consumption due to the smallest eigenvalue [34]. In such a case, we do not achieve any receive diversity. The sum of all streams for all the users is equal to $\sum_{k=1}^{U} L_{k}=N_{t}$.

As the diagonal matrix $\Sigma_{k}$ is obtained by $\widetilde{\Lambda}_{k} \boldsymbol{\Phi}_{k}$, the equal gain decomposition described in Section 2.3 can be applied as

$$
\overline{\boldsymbol{H}}_{k}=\left[\begin{array}{c}
\sum_{k} \\
\sqrt{\alpha} \boldsymbol{I}_{N}
\end{array}\right]=\boldsymbol{Q}_{k} \boldsymbol{R}_{k} \boldsymbol{P}_{k}^{*}, \quad 1 \leq k \leq U
$$

$\mathbf{Q}_{k}$ is an unitary matrix and the upper triangular matrix $\mathbf{R}_{k}$ has the equal eigenvalues $\bar{r}=$ $\bar{\sigma}=\left(\Pi_{k=1}^{N_{t}} \sqrt{\sigma_{k}^{2}+\alpha}\right)^{1 / N_{t}}$. The power loading matrix $\mathbf{P}$ is derived through UCD method, and the first $L_{k}$ columns of $\mathbf{Q}_{k}$ is used for decoder postcoder of user $k$. This process is clearly capacity lossless from the following Weyl's multiplicative majorization conditions.

Theorem 1: Let $\mathbf{A}$ be a matrix with rank $n$. Let the ordered eigenvalues and singular values of $\mathbf{A}$ be $\lambda_{1}, \cdots, \lambda_{n}$ and $\sigma_{1}, \cdots, \sigma_{n}$, respectively. Then for $1 \leq k \leq n$,

$$
\left|\lambda_{1}\right| \ldots\left|\lambda_{k}\right| \leq \sigma_{1} \ldots \sigma_{k}, \quad\left|\lambda_{1}\right| \ldots\left|\lambda_{n}\right| \leq \sigma_{1} \ldots \sigma_{n} .
$$

Proof: See [27, 35].

From Theorem 1, we see that the diagonal element of $\mathbf{R}$ in (9) is the geometric mean of singular value of $\mathbf{H}$ [27]. The permutation matrix for the $k$ th user $\mathbf{P}_{k}$ is an orthonormal matrix, and it can be achieved by QR decomposition. By applying Givens $2 \times 2$ rotation process up to $L_{k}$, we obtain a complete set of the precoding matrix $\mathbf{Q}_{k}, \mathbf{R}_{k}$ and $\mathbf{P}_{k}$ in (9). One important point of $\mathbf{Q}_{k}$ is that it starts with $\widetilde{\mathbf{V}}_{k}^{0} \mathbf{V}_{k}^{1}$, not $\boldsymbol{V}_{k}$, to preserve zero interference criterion. The first $L_{k}$ columns of $\mathbf{Q}_{k}$ is the decoder postcoder vector of user $k, \mathbf{W}_{k}$.

After applying block-wise equal channel gain decomposition for every user, we can write $N_{t} \times N_{t}$ power loading matrix $\mathbf{P}$ as $\mathbf{P} \triangleq \operatorname{diag}\left\{\mathbf{P}_{1}, \mathbf{P}_{2}, \cdots, \mathbf{P}_{U}\right\}$, and consequently have the precoder $\mathbf{F}=\widetilde{\mathbf{V}} \boldsymbol{\Phi} \mathbf{P}$. Given the precoder $\mathbf{F}$, the $k$ th user's effective channel $\overline{\mathbf{H}}_{k}=\mathbf{W}_{k}^{*} \widetilde{\mathbf{H}}_{k} \mathbf{F}$ is obtained. The $k$ th user's decoder combining matrix $\boldsymbol{W}_{k}^{*}$ is given to each decoder as a decoder postcoder. Then the input-output relation of our two-step precoding is finally given by

$$
\mathbf{y}_{k}=\mathbf{W}_{k}^{*} \widetilde{\mathbf{H}}_{k} \overline{\mathbf{V}} \Phi \mathbf{P x}+\mathbf{W}_{k}^{*} \mathbf{n}=\sum_{k} \mathbf{P}_{k} \mathbf{x}_{L_{x}}+\widetilde{\mathbf{n}} .
$$

The joint precoder and postcoder design in (10) shows that we may generate an effective channel with equal channel gain without causing interference between users. Our next step is about sum rate capacity and diversity analysis, which are two measurements for the throughput and reliability performance in the MIMO channel. 


\subsection{Sum Rate Capacity Analysis}

In this chapter, the sum rate capacity of our proposed algorithm is analyzed. Recall that the received signal for the $k$ th user in (10) is

$$
\mathbf{y}_{k}=\sum_{k} \mathbf{P}_{k} \mathbf{x}_{L_{x}}+\widetilde{\mathbf{n}}_{k} .
$$

If we let $\mathbf{U}_{x}$ be input covariance matrix and $\mathbf{U}_{k}$ be noise covariance matrix for $k$ th user's effective noise $\widetilde{\mathbf{n}}_{k}$, then the mutual information for $k$ th user can be written as

$$
l_{k}=\log _{2} \operatorname{det}\left(\boldsymbol{I}_{k}+\sum_{k} \boldsymbol{P}_{k} \boldsymbol{U}_{x}\left(\sum_{k} \boldsymbol{P}_{k}\right)^{*} \boldsymbol{U}_{k}^{-1}\right) .
$$

Because we maintain zero-interference criterion, the aggregate mutual information for $U$ users is equal to the sum of independent mutual information per user. Also noise covariance matrix $\mathbf{U}_{k}$ is the same to that of AWGN channel because multiplication of unitary postcoder $\mathbf{W}^{*}$ at the decoder does not change the second order statistics of the noise. Therefore, the total sum rate with $U$ users is given by

$$
l_{\{1, \cdots, U\}}=\sum_{k=1}^{U} l_{k}=\log _{2} \operatorname{det}\left(\mathbf{I}_{N_{t} \times N_{t}}+\frac{\Sigma \mathbf{P} \mathbf{U}_{x}(\Sigma \mathbf{P})^{*}}{\sigma_{n}^{2}}\right) .
$$

The maximum sum rate is the mutual information maximizer with given input power constraint, $\operatorname{tr}\left\{\boldsymbol{U}_{x}\right\} \leq P_{T}$. The power allocation matrix $\mathbf{P}$ is normalized since it is a unitary matrix. Therefore, the achievable sum rate throughput is

$$
C=\max _{\left\{\mathbf{U}_{x}: \operatorname{tr}\left\{\mathbf{U}_{x}\right\} \leq P_{T}\right\}} l_{\{1, \cdots, U\}}=\max _{\left\{\mathbf{U}_{x}: \operatorname{tr}\left\{\mathbf{U}_{x}\right\} \leq P_{T}\right\}} \sum_{k=1}^{U} l_{k} \stackrel{(a)}{=} \log _{2} \operatorname{det}\left(\mathbf{I}+\frac{\Sigma^{2}}{\sigma_{n}^{2}}\right) .
$$

The equality (a) holds by the determinant identity $\operatorname{det}(\mathbf{I}+\mathbf{A B})=\operatorname{det}(\mathbf{I}+\mathbf{B} \mathbf{A})$ and capacity lossless property in Theorem 1. Choosing a proper power loading parameter to maximize the channel capacity is equivalent to the standard waterfilling process in [30] and therefore, the sum capacity of our algorithm is equivalent to the block diagonalization with waterfilling solution in [16]. When the number of streams per user goes one, we still achieve the same sum rate throughput, but diversity gain will be decreased as shown in the following section.

\subsection{Diversity Gain Analysis}

Diversity gain is a tool to measure error rate decay per SNR improvement. Unlike to the openloop MIMO literature where no CSIT is available at the transmitter [36], diversity analysis in closed-loop MIMO is closely related to the transmit power optimization since one can adjust transmit power or transmission rate to prevent error outage event. In case where the data rate is fixed, the diversity analysis is a good measure to see the error rate performance in closedloop literature.

Let us derive an expression for the probability of outage $P_{e}(\rho)$ when one is attempting to communicate at rate $r \log (\rho)$ bits/channel use, where $r$ is known as spatial multiplexing gain and $\rho$ is SNR. To make our analysis simple, we assume that the target SNR per user is identical and is simply achieved by proper power allocation. For fixed AWGN channel matrix $\mathbf{H}$, we can formulate the error exponent using sum rate derived in the previous chapter as

$$
P_{e}(\rho)=\operatorname{Pr}\left(\log \operatorname{det}\left(\mathbf{I}+\rho \sum^{2} \mathbf{P}^{2}\right)<r \log (\rho)\right) .
$$


From the results of previous section, we know that the sum capacity is achieved when appropriate power is allocated to achieve given SNR requirement. Therefore, we have the following inequality

$$
P_{e}(\rho) \leq \operatorname{Pr}\left(\log \left(\prod_{k=1}^{U}\left(1+\rho \lambda_{k} \mathbf{P}_{k}\right)^{L_{k}}\right)<r \log (\rho)\right)
$$

where $\lambda_{k}$ denotes the $k$ th user's eigenvalue. Because of the equal channel gain decomposition, user $k$ has $L_{k}$ identical eigenvalues $\lambda_{k}$. Then the outage corresponds to the event $\log \left(\prod_{k=1}^{U}\left(1+\rho \lambda_{k}\right)^{L_{k}}\right)<r \log (\rho)$ is equivalent to

$$
\prod_{k=1}^{U}\left(1+\rho \lambda_{k}\right)^{L_{k}}<\rho^{r} .
$$

In (17), the $U$ independent eigenvalues $\lambda_{1}, \cdots, \lambda_{U}$ corresponding to each user can be written as the product of independent Chi-squared distribution $\chi$, each with $2\left(N_{t}-N_{r, k}+1\right)$ degrees of freedom, under the high SNR assumption [34, 37]. By applying $\lambda_{i}=\rho^{-\alpha_{i}}$ to (17) and high SNR approximation yield the following outrage probability

$$
P_{e}(r \log \rho)=\operatorname{Pr}\left(\prod_{i=1}^{U}\left(1+\rho \lambda_{i}\right)^{L_{i}}<\rho^{r}\right)=\operatorname{Pr}\left(\sum_{i}^{U}\left(\left(1-\alpha_{i}\right) L_{i}\right)^{+}<r\right) .
$$

Our next interest is to formulate of distribution function of the random vector $\lambda=\left[\lambda_{1}, \cdots\right.$, $\lambda_{U}$ ]. According to [36] Lemma 3] and independent Chi-squared distribution property, the joint pdf of random vector $\lambda$ is

$$
P(\lambda)=A \prod_{i=1}^{U} \lambda_{i}^{L_{i}\left(N_{t}-N_{r, i}\right)} e^{-\sum_{i=1}^{U} \lambda_{i}^{L_{i}}} \prod_{j>i}\left(\lambda_{i}-\lambda_{j}\right)^{2} .
$$

By applying (18) into (19) with the outage event $\varepsilon=\left\{\lambda: \sum_{\alpha}\left(\left(1-\lambda_{i}\right) L_{i}\right)^{+}<r\right\}$ yields

$$
P_{e}(r \log \rho)=\int_{\varepsilon} p(\alpha) d \alpha=\int_{\varepsilon} \prod_{i=1}^{U} \rho^{-\left(2 i-1-\left|N_{t}-N_{r, i}\right|\right) L_{i} \alpha_{i}} d \alpha .
$$

From the above equation, it is enough to determine the dominating exponent for the diversity gain. The diversity gain of algorithm can be written as

$$
\begin{aligned}
d\left(r^{\prime}\right) & =-\lim _{\rho \rightarrow \infty} \frac{\log P_{e}(\rho)}{\log \rho} \\
& =-\lim _{\rho \rightarrow \infty} \frac{\log \int_{\varepsilon} \prod_{i=1}^{U} \rho^{-\left(2 i-1-\left|N_{t}-N_{R, i}\right|\right) L_{i} \alpha_{i}} d \alpha}{\log \rho} \\
& =\inf _{\alpha_{i}} \sum_{i=1}^{U} L_{i} \alpha_{i}\left(2 i-1+\left|N_{r, k}-N_{t}\right|\right) .
\end{aligned}
$$

Equation (23) shows that when trying to communicate with rate $r^{\prime}$, i.e., $r=r^{\prime}$, the 
maximum diversity order (inf is achieved when $\alpha_{1}=\cdots=\alpha_{U-r^{\prime}}=1[36]$ ) is

$$
\begin{aligned}
d\left(r^{\prime}\right) & =\sum_{i=1}^{U-r^{\prime}} L_{i}\left(2 i-1+\left|N_{r, i}-N_{t}\right|\right) \\
& =\sum_{i=1}^{U-r^{\prime}} L_{i}(2 i-1)+\left(U-r^{\prime}\right)\left(\left|N_{r, i}-N_{t}\right|\right) .
\end{aligned}
$$

The results in (25) state that when there is $N_{t}$ single antenna users, full diversity order $\left(N_{t}\right.$ $\left.-r^{\prime}\right)\left(N_{r}-r^{\prime}\right)$ is achieved when attempting to communicate at rate $r^{\prime}\left(r^{\prime} \leq \min \left\{N_{t}, N_{r}\right\}\right)$. If four transmit antennas and two users with two decoder antennas are considered, the maximum achievable rate is $2\left(2-r^{\prime}\right)\left(4-r^{\prime}\right)$, when attempting to communicate at $r^{\prime}$. This shows that our scheme achieves full receive diversity gain while the maximum multiplexing gain per user cannot exceed $R \leq \min \left(N_{t}, N_{r, k}\right)$. The numerical results in Section 4 show that our algorithm achieves full diversity gain in given data rate.

\subsection{Receiver and Feedback Strategies}

In this section, the decoding methods and feedback strategies for the proposed algorithm is analyzed. We assume that the postcoding matrix per user derived in Section 3.1 is given to each decoder by a proper signaling. The result in (10) shows that the composite effective channel matrix is block diagonalizable per user. The $k$ th user has $N_{r, k} \times N_{t}$ channel with real channel elements, without multiuser interference caused by other users. Since the effective channel is real, we may decode real part and imaginary part of the transmit symbols independently. Hence, the following per symbol ML metric can be applied as

$$
\hat{s}_{k}=\arg \min _{s_{k} \in C}\left\|\boldsymbol{y}_{k}-\sum_{k} \boldsymbol{P}_{k} s_{k}\right\|^{2}
$$

for real and imaginary part of $\hat{s}_{k}$, independently. The decoding complexity is also reduced by the independent decoding, while achieving full receive diversity as analyzed in Section 3.3.

Another advantage of the proposed algorithm is on the feedback signaling for link adaptation at the transmitter. Unlike to the linear decoder such as ZF and MMSE, SINR after ML decoding cannot be explicitly calculated, to our best knowledge. Even though there is an exhaustive search algorithm based on pairwise error probability (PEP) [38], it is too complex and not analogous to multiuser MIMO with link adaptation. As the modulation and coding selection (MCS) per streams is not equal within each user, different modulation order results in different PEP in the multiuser MIMO link. But per stream link adaptation is not required in our algorithm, since the effective channel gain is already equalized for all streams within a user. We may apply link adaptation per user, not per stream, using per user SINR.

The problem to generate effective SINR become simpler since the eigenvalues within each user are equal. The union bound for coded binary transmission and ML decoding can be written as

$$
P_{e}(\gamma) \leq \sum_{d=d_{\min }}^{\infty} \alpha_{d} P_{2}(d, \gamma)
$$


where $\gamma$ is effective SINR that we want to derive, $\alpha_{d}$ is the number of codewords with hamming weight $d$, and $\mathrm{P}_{2}(d, \gamma)$ is PEP with respect to hamming distance $d$ and effective SINR $\gamma$. Then the PEP upper bound is obtained using Chernoff bound

$$
P_{e}(\gamma) \leq \sum_{d=d_{\min }}^{\infty} \alpha_{d} P_{2}(d, \gamma)<\sum_{d=d_{\min }}^{\infty} \alpha_{d}\left[P_{2}(1, \gamma)\right]^{d} \triangleq P_{e, \text { Chernoff }}(\gamma)
$$

From (28), the Chernoff-bounded error probability only depends on the weight distribution of the code and the Chernoff-bounded symbol error probability. If different SINR values are used, (28) may be solved as the Union Chernoff bound for multiple-state channel [39], consequently requires a weighting parameter to adjust the effective SINR to a specific combination of modulation and coding rate. The proposed algorithm makes the work simpler, since multiple identical eigenvalues may reduce the computation from multi-state Chernoff bound to single-state bound. Thus, we have a simple effective SINR for $k$ th user as

$$
\gamma_{k, e f f}=-\beta_{M L} \ln \left(\frac{1}{L_{k}} \sum_{i=1}^{L_{k}} \exp \left(-\frac{\gamma_{k, i}}{\beta_{M L}}\right)\right) \approx-\beta_{M L} \ln \left(\exp \left(-\frac{\bar{\gamma}_{k}}{\beta_{M L}}\right)\right)=\bar{\gamma}_{k}
$$

where $\bar{\gamma}_{k}$ is the received SINR at user $k$. From (29), we can use effective SINR measured at the decoder before applying ML decoding as an indicator for the link adaptation to the transmitter. If limited feedback is applied as in [14], our strategy is simply feedback the SINR value measured at the decoder, or as a form of MCS indicator by quantizing the channel capacity as

$$
Q\left(C_{k}\right)=\left\lfloor C_{k}\right\rfloor \leq L_{k} \log _{2}\left(1+\bar{\gamma}_{k}\right)
$$

where $[\cdot]$ indicates lower bound in quantization.

Another advantage of the proposed algorithm is on the system level performance evaluation The effective SINR is used to map the instantaneous channel state information into a specific MCS table satisfying target error rate. It has been a hard problem to obtain per stream effective SINR when ML decoding is used, resulting in performance leakage due to the effective SINR mismatch. Using the proposed methods, each user can generate one representative effective SINR without mismatch. The proposed strategy is the simplest form of the exponential effective signal to interference ratio mapping (EESM) for ML decoder so far, to our best knowledge.

\section{Simulation Results}

In this section, numerical simulation results for the candidate algorithm are presented. We first compare our algorithm with previously proposed MIMO schemes in Table 1. To illustrate sum rate performance, we present sum rate throughput in various SNR regions in Fig. 2. Error rate performance is presented in Fig. 3 and Fig. 4 to see the diversity gain performance of the proposed algorithm. 
Table 1. Comparison of various single-user and multiuser MIMO algorithms

\begin{tabular}{|c|c|c|c|c|c|}
\hline & SVD (Ideal) & OBF & BD & UCD & Proposed \\
\hline \hline Precoder & $\boldsymbol{V}$ & $Q(\boldsymbol{V})$ & {$\left[\widetilde{\mathbf{V}}_{1}^{0} \mathbf{V}_{1}^{1} \cdots \widetilde{\mathbf{V}}_{U}^{0} \mathbf{V}_{U}^{1}\right]$} & $\boldsymbol{V} \boldsymbol{\Phi P}$ & $\overline{\mathbf{V}} \boldsymbol{\Phi} \boldsymbol{P}$ \\
\hline $\begin{array}{c}\text { Receive } \\
\text { Filter }\end{array}$ & $\boldsymbol{U}^{*}$ & - & $\boldsymbol{U}^{*}$ & $\widetilde{\mathbf{Q}}_{u}^{*}$ & $\overline{\mathbf{Q}}^{*}$ \\
\hline $\begin{array}{c}\text { Effective } \\
\text { Channel }\end{array}$ & Diagonal & Near orthogonal & Block diagonal (BD) & Upper triangular & Uniform BD \\
\hline $\begin{array}{c}\text { Multiuser or } \\
\text { Single user }\end{array}$ & $\mathrm{SU}$ & $\mathrm{MU}$ & $\mathrm{MU}$ & $\mathrm{SU}$ & $\mathrm{MU}$ \\
\hline $\begin{array}{c}\text { Decoding } \\
\text { Method }\end{array}$ & - & ZF, MMSE & - & SIC, THP & Single MLD \\
\hline $\begin{array}{c}\text { Achievable } \\
\text { Sum Rate }\end{array}$ & $C$ & $<C$ & $\approx C$ & $C$ & $\approx C$ \\
\hline $\begin{array}{c}\text { Diversity } \\
\text { Gain }\end{array}$ & $N_{t} N_{r, k}$ & $\min \left\{N_{t}, N_{r, k}\right\}$ & $\min \left\{N_{t}, N_{r, k}\right\} \times N_{r, k}$ & $N_{t} \times \min \left\{N_{r, k}\right\}$ & $N_{t} N_{r, k}$ \\
\hline $\begin{array}{c}\text { SINR } \\
\text { Feedback }\end{array}$ & High & Medium & Medium & Low & Low \\
\hline Complexity & High & Low & Low & Medium & Medium \\
\hline
\end{tabular}



Fig. 2. Sum rate performance comparison between DPC, BD, ZF (channel inversion) and the proposed method for $(2,2,4)$ case -4 transmit antennas, 2 users with 2 receive antennas 
Fig. 2 shows the sum rate performance of the proposed algorithm. For four transmit antenna and two users with two receive antenna each, our algorithm shows a good performance compared to conventional ZF precoding methods. Also, the achievable rate region of our algorithm is almost the same to the BD achievable region. This is because our algorithm perfectly cancels the multiuser interference, while maintaining efficient power loading per user. We assume that the number of aggregated receive antennas for all users are equal to the transmit antenna, so no scheduling gain is affected to the performance. If we have more users and thus can be benefited by the multiuser diversity gain (or equivalently scheduling gain), the performance gap between DPC and our proposed algorithm may be shortened.

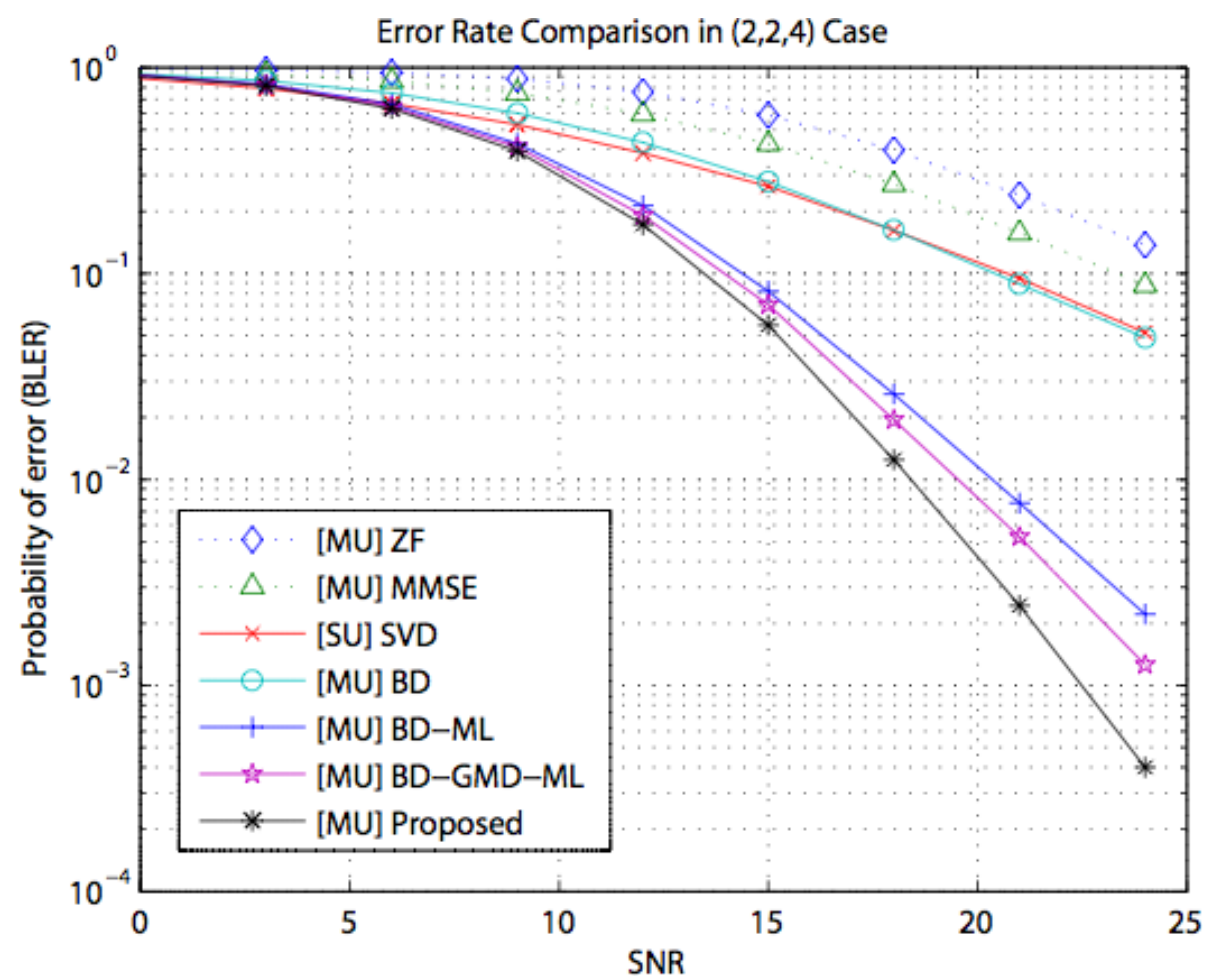

Fig. 3. Block error rate performance comparison between ZF (channel inversion), MMSE, SVD without power optimization, BD, BD with ML decoder, BD-GMD with ML decoder and the proposed algorithm in $(2,2,4)$ configuration -4 transmit antennas, 2 users with 2 receive antennas

Fig. 3 and Fig. 4 show the block error rate performance of the proposed algorithm. We assume four transmit antenna and two users with two receive antennas in Fig. $\mathbf{3}$ and compare the proposed algorithm with ZF, MMSE, SVD, BD, BD with ML and BD-GMD with ML decoder. Conventional BD achieves diversity one as ZF and MMSE, but our proposed algorithm achieves diversity order four. It is better than BD-GMD with ML decoder and BD with ML decoder because UCD achieves full diversity as proven in Section 3.3.

Fig. 4 shows the diversity performance in different antennas configurations. We assume six transmit antennas and two different receive antenna configurations: 3 users with 2 decoder antennas and 2 users with 3 receive antennas. As we analyzed in Section 3.1, configurations with more receive antenna is advantageous in achieving more diversity gain. The simulation results in Fig. 4 reinforce the analysis in Section 3.1 and 3.3. 


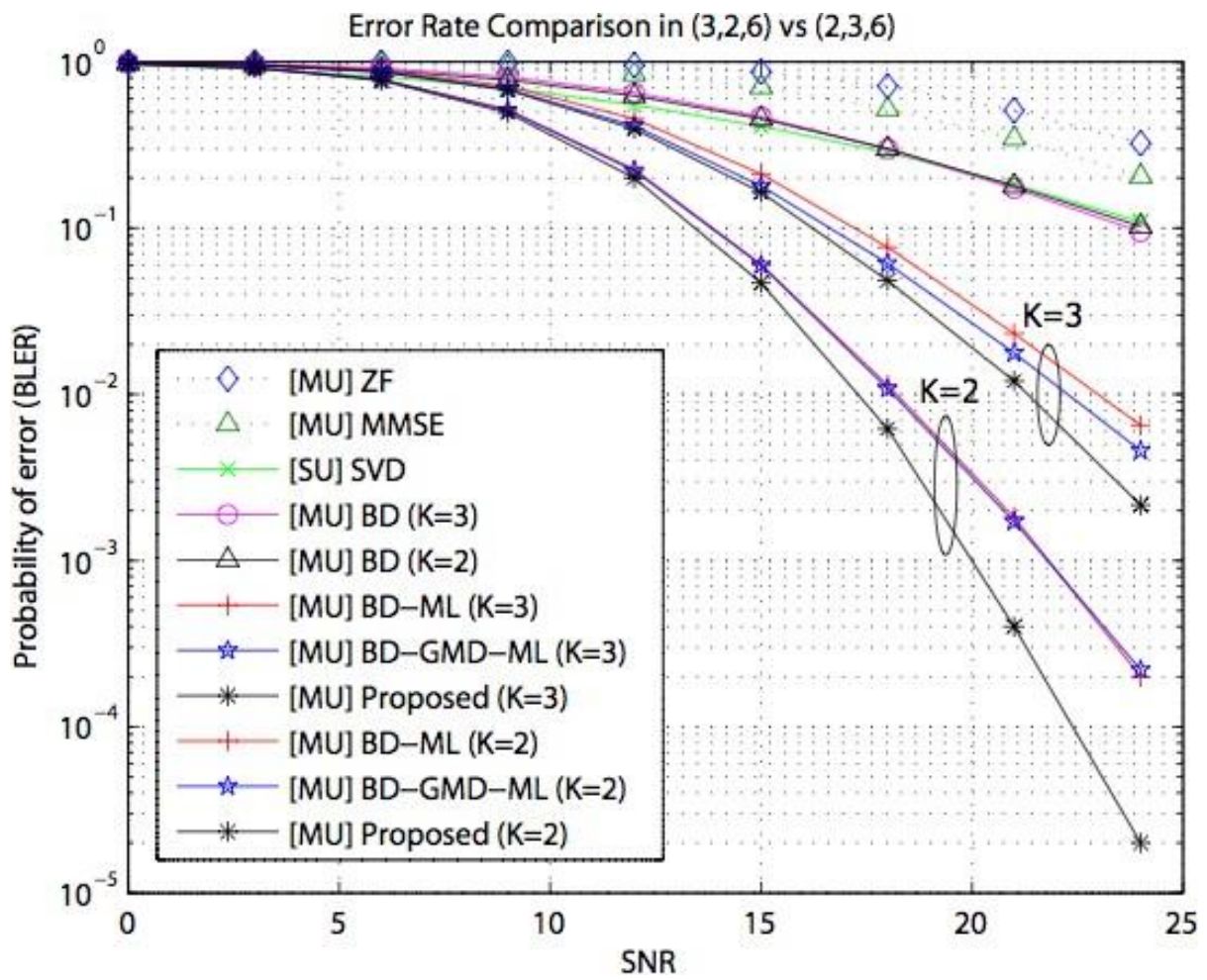

Fig. 4. Block error rate performance comparison in two antenna configurations $(3,2,6)$ and $(2,3,6)-6$ transmit antennas and 3 users with 2 receive antennas vs 6 transmit antennas and 2 users with 3 receive antennas

We compare the proposed algorithm with some previously proposed algorithms in Table 1. The reference algorithms are SVD, OBF [14], BD [16] and UCD [28], which are wellknown closed-loop MIMO algorithms. We investigate the difference in transmit precoder design, receive filter, effective channel shape, multiuser diversity, decoding method if there is no feed-forward information, achievable sum rate, diversity gain, SINR feedback requirement and finally computational complexity. Table 1 shows that the proposed method has a good tradeoff between complexity and performance. It requires slightly more complexity than BD and UCD but achieves a good sum rate performance and optimal diversity gain. SINR feedback reduction even with ML decoding using single-stage Chernoff bound is another merit of the proposed algorithm.

\section{Conclusion}

In this paper, a new multiuser MIMO precoder and postcoder design method is proposed. The proposed method uses two step precoding by combining equal rate decomposition under with multiuser interference criterion. We proved that the proposed algorithm can be easily combined with a symbol decodable MLD and can generate simple but accurate effective SINR. We showed that the algorithm achieved good sum rate performance and full diversity gain. Comparison with other algorithms in both SU and MU MIMO was also presented.

We note that the scheduling algorithm of our proposed algorithm is still under investigation. Because diversity order per user is upper bounded by the number of receive antenna per user, the scheduling strategy in terms of maximizing diversity gain is to schedule users with more 
receive antennas. But when the number of users is high, it is easy to schedule users with single streams, to meet the zero-interference criterion. A qualitative analysis between precoding and beamforming based on the number of users in the scheduling queue will be another interesting research area.

\section{References}

[1] D. Gesbert, M. Kountouris, R. W. Heath Jr., C. B. Chae, and T. Sa"lzer, "From single user to multiuser communications: Shifting the MIMO paradigm," IEEE Signal Processing Magazine, vol. 24, no. 5, pp. 36-46, 2007. Article (CrossRef Link)

[2] G. Caire and S. Shamai, "On the achievable throughput of a multi-antenna Gaussian broadcast channel," IEEE Transactions on Information Theory, vol. 49, no. 7, pp. 1691-1706, July 2003. Article (CrossRef Link)

[3] H. Weingarten, Y. Steinberg, and S. Shamai, "The capacity region of the Gaussian multiple-input multiple-output broadcast channel," IEEE Transactions on Information Theory, vol. 52, no. 9, pp. 3936-3964, Sep. 2006. Article (CrossRef Link)

[4] A. B. Nogueras, A. Davoodi, P. Kerret, D. Gesbert, N. Gresset, and S. Jafar, "DoF Region of the Decentralized MIMO Broadcast Channel—-How many informed antennas do we need?," in Proc. of 2020 IEEE International Symposium on Information Theory, pp. 1468-1473, 2020.

Article (CrossRef Link)

[5] K. Kim and J. Lee, "Deep CNN based pilot allocation scheme in massive MIMO systems," KSII Transactions on Internet and Information Systems, vol. 14, no. 10, pp. 4214-4230, 2020.

Article (CrossRef Link)

[6] T. Yoo and A. Goldsmith, "On the optimality of multiantenna broadcast scheduling using zeroforcing beamforming," IEEE Journal on Selected Areas in Communications, vol. 24, no. 3, pp. 528-541, Mar. 2006. Article (CrossRef Link)

[7] W. Xu and M. Lin, "Downlink Capacity Analysis of Distributed Antenna Systems with Imperfect Channel State Information," KSII Transactions on Internet and Information Systems, vol. 11, no. 1, pp. 253-271, 2017. Article (CrossRef Link)

[8] N. Jindal and A. Goldsmith, "Dirty-paper coding versus tdma for MIMO broadcast channels," IEEE Transactions on Information Theory, vol. 51, no. 5, pp. 1783-1794, May 2005.

Article (CrossRef Link)

[9] J. Cao, S. Yu, J. Yang, Y. Zhang, and S. Zhao, "Massive MIMO two-hop relay systems over Rician fading channels," KSII Transactions on Internet and Information Systems, vol. 13, no. 11, pp. 5410-5426, 2019. Article (CrossRef Link)

[10] S. Vishwanath, N. Jindal, and A. Goldsmith, "Duality, achievable rates, and sum capacity of Gaussian MIMO broadcast channels," IEEE Transactions on Information Theory, vol. 49, no. 10, pp. 2658-2668, Aug. 2003. Article (CrossRef Link)

[11] X. Li, L. Li, and L. Xie, "Achievable sum rate analysis of ZF receivers in 3D MIMO systems," KSII Transactions on Internet and Information Systems, vol. 8, no. 4, pp. 1368-1389, 2014. Article (CrossRef Link)

[12] X. Yunjia, H. Kaizhi, H. Xin, Z. Yi, C. Yajun, and J. Wenyu, "CoMP Transmission for Safeguarding Dense Heterogeneous Networks with Imperfect CSI," KSII Transactions on Internet and Information Systems, vol. 13, no. 1, pp. 110-132, 2019. Article (CrossRef Link)

[13] D. Gore, R.W. Heath, and A. Paulraj, "Transmit selection in spatial multiplexing systems," IEEE Communications Letters, vol. 6, no. 11, pp. 491-493, Nov. 2002. Article (CrossRef Link)

[14] K. Huang, R. W. Heath, Jr., and J. G. Andrews, "Performance of orthogonal beamforming for SDMA with limited feedback," IEEE Transations on Vehicular Technology, vol. 58, no. 1, pp. 152-164, 2009. Article (CrossRef Link)

[15] C. Yuqing, L. Xiaoyan, S. Xixia, and P. Su, "Resource allocation in multi-user MIMO-OFDM systems with double-objective optimization," KSII Transactions on Internet and Information Systems, vol. 12, no. 5, pp. 2063-2081, 2018. Article (CrossRef Link) 
[16] Q. Spencer, A. Swindlehurst, and M. Haardt, "Zero-forcing methods for downlink spatial multiplexing in multiuser MIMO channels," IEEE Transactions on Signal Processing., vol. 52, no. 2, pp. 461-471, Feb. 2004. Article (CrossRef Link)

[17] L. U. Choi and R. D. Murch, "A transmit preprocessing technique for multiuser MIMO systems using a decomposition approach," IEEE Transactions on Wireless Communications, vol. 3, no. 1, pp. 20-24, Jan. 2004. Article (CrossRef Link)

[18] C. B. Peel, B. M. Hochwald, and A. L. Swindlehurst, "A vector-perturbation technique for near capacity multiantenna multiuser communication - part I: channel inversion and regularization," IEEE Transactions on Communications, vol. 53, no. 1, pp. 195-202, Jan. 2005.

Article (CrossRef Link)

[19] C. Windpassinger, R. F. H. Fischer, T. Vencel, and J. B. Huber, "Precoding in multiantenna and multiuser communications," IEEE Transactions on Wireless Communications, vol. 3, no. 4, pp. 1305-1316, July 2004. Article (CrossRef Link)

[20] B. M. Hochwald, C. B. Peel, and A. L. Swindlehurst, "A vector-perturbation technique for near capacity multiantenna multiuser communication - part II: perturbation," IEEE Transactions on Communications, vol. 53, no. 3, pp. 537-544, Mar. 2005. Article (CrossRef Link)

[21] U. Fincke and M. Pohst, "Improved methods for calculating vectors of short length in a lattice, including a complexity analysis," Mathematics of Computation, vol. 44, pp. 463-471, 1985. Article (CrossRef Link)

[22] N. Yoo, J. Back, H. Choi, and K. Lee, "ML Symbol Detection for MIMO Systems in the Presence of Channel Estimation Errors," KSII Transactions on Internet and Information Systems, vol. 10, no. 11, pp. 5305-5321, 2016. Article (CrossRef Link)

[23] B. Hassibi and H. Vikalo, "On the sphere-decoding algorithm I: Expected complexity," IEEE Transactions on Signal Processing, vol. 53, no. 8, pp. 2806-2818, Aug. 2005. Article (CrossRef Link)

[24] K. Higuchi, H. Kawai, N. Maeda, and M. Sawahashi, "Adaptive selection of surviving symbol replica candidates based on maximum reliability in QRM-MLD for OFCDM MIMO multiplexing," in Proc. of IEEE Global Telecommunications conference, vol. 4, pp. 2480-2486, vol. 4, 2004. Article (CrossRef Link)

[25] J. Kim, J. Kang, and W. Y. Yang, "Recursive decoding for OFDM systems with multiple transmit antennas," IEICE Transactions on Communications, vol. 88, no. 6, pp. 2664-2667, June 2005. Article (CrossRef Link)

[26] J. Jalden, L. Barbero, B. Ottersten, and J. Thompson, "Full diversity detection in MIMO systems with a fixed-complexity sphere decoder," in Proc. of IEEE International Conference Acoustics, Speech and Signal Processing, vol. 3, pp. III-49-III-52, Apr. 2007. Article (CrossRef Link)

[27] Y. Jiang, J. Li, and W. Hager, "Joint transceiver design for MIMO communications using geometric mean decomposition," IEEE Transactions on Signal Processing, vol. 53, no. 10, pp. 3791-3803, Oct. 2005. Article (CrossRef Link)

[28] Y. Jiang, J. Li, and W. Hager, "Uniform channel decomposition for MIMO communications," IEEE Transactions on Signal Processing, vol. 53, no. 11, pp. 4283-4294, Nov. 2005. Article (CrossRef Link)

[29] J. Lee and N. Jindal, "High SNR analysis for MIMO broadcast channels: Dirty paper coding versus linear precoding," IEEE Transactions on Information Theory, vol. 53, no. 12, pp. 47874792, Dec. 2007. Article (CrossRef Link)

[30] E. Telatar, "Capacity of multi-antenna Gaussian channels," European Transactions Telecommunications, vol. 10, pp. 585-595, Nov. 1999. Article (CrossRef Link)

[31] S. Lin, W. W. L. Ho, and Y.C. Liang, "Block-diagonal geometric mean decomposition (BD-GMD) for multiuser MIMO broadcast channels," IEEE Transactions on Wireless Communications, vol. 7, no, 7 pp. 2778-2789, 2009. Article (CrossRef Link)

[32] S. Lin, W. W. L. Ho, and Y. C. Liang, "MIMO broadcast communications using block-diagonal uniform channel decomposition (BD-UCD)," in Proc. of 2006 IEEE 17th International Symposium on Personal, indoor and Mobile Radio Communications, pp. 1-5, Sep. 2006. Article (CrossRef Link) 
[33] K. J. Lee, B. M. Hochwald, and I. Lee, "Blockwise uniform channel decomposition for MIMO systems," in Proc. of Globecom 2007, IEEE Global Telecommunications Conference, pp. 31543158, Nov. 2007. Article (CrossRef Link)

[34] A. Edelman, "Eigenvalues and condition number of random matrices," Ph.D. dissertation, Dept. Mathematics, MIT, Cambridge, MA, 1989.

[35] H. Weyl, "Inequalities between the two kinds of eigenvalues of a linear transformation," National Academy of Sciences of the United States of America, vol. 35, pp. 408-411, 1949. Article (CrossRef Link)

[36] L. Zheng and D. N. C. Tse, "Diversity and multiplexing: a fundamental tradeoff in multipleantenna channels," IEEE Transactions on Information Theory, vol. 49, no. 5, pp. 1073-1096, May 2003. Article (CrossRef Link)

[37] T. Anderson, "An Introduction to Multivariate Statistical Analysis," 3rd ed., Wiley, 1958.

[38] H. Liu, L. Cai, H. Yang, and D. Li, "EESM based link error prediction for adaptive MIMO-OFDM system," in Proc. of 2007 IEEE $66^{\text {th }}$ Vehicular Technology Conference, pp. 559-563, Apr. 2007. Article (CrossRef Link)

[39] Ericsson, "System level evaluation of OFDM - further considerations," TSG-RAN WG1 \#35, R1031303, Nov. 2003. 


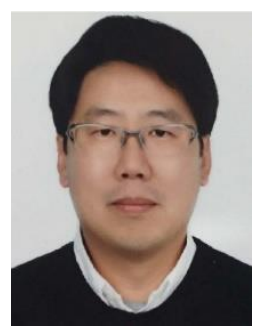

Insoo Hwang received the B.S. degree in electronics and electrical engineering from the Pohang University of Science and Technology, Pohang, South Korea, the M.S. degree in electrical engineering from the University of Southern California at Los Angeles, Los Angeles, and the Ph.D. degree in electrical and computer engineering from The University of Texas at Austin, Austin. At Qualcomm Research, he is working with the next-generation cellular systems and standards, focusing on in-device coexistence and large-scale, densely deployed cooperative heterogeneous and small cell networks.

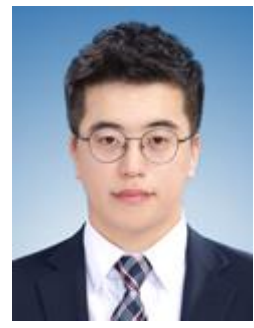

Inseok Kang received the B.S. and M.S degrees in Department of Information and Communications Engineering, Myongji University, South Korea, respectively. He is currently with Qucell Networks Inc., with a focus on LTE cellular systems and standards, and Femto-cell research. He is currently interested in the 5G mobile communication systems, small cell test solution, and optimization solutions for the design goals and actual quality after establishment of the communication network.

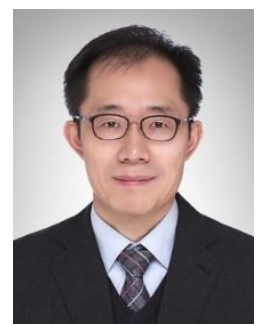

Intae Hwang received a B.S. degree in Electronics Engineering from Chonnam National University, Gwangju, Korea in 1990 and a M.S. degree in Electronics Engineering from Yonsei University, Seoul, Korea in 1992, and a Ph.D. degree in Electrical \& Electronics Engineering from Yonsei University, Seoul, Korea in 2004. He was a senior engineer at LG Electronics from 1992 to 2005. He is currently a Professor in the Department of Electronic Engineering and Department of ICT Convergence System Engineering at Chonnam National University, Gwangju, Korea from 2006. His research interests include digital communication, wireless communication system, and next-generation mobile communication: MIMO-OFDM, V2X, NR-MIMO, NTN, and artificial intelligence.

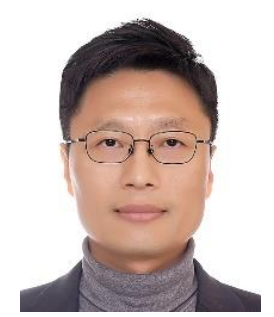

Cheolwoo You received the B.S., M.S., and Ph.D. degrees in Electronics Engineering from Yonsei University, Seoul, South Korea, in 1993, 1995, and 1999, respectively. From January 1999 to April 2003, he worked as a Senior Research Engineer with the LG Electronics, Gyeonggi, South Korea. From August 2004 to July 2006, he was with the Samsung Electronics, Suwon, South Korea. He is currently a Professor in the Department of Information and Communications Engineering, Myongji University, South Korea. He is currently interested in the 5th generation communication systems, deep learning, and signal processing. 